\title{
MOOC M4D: An Overview and Learner's Viewpoint on Autumn 2013 Course
}

\author{
http://dx.doi.org/10.3991/ijim.v8i1.3486 \\ Maitrayee Ghosh \\ Florida State University, Tallahassee, FL, USA
}

\begin{abstract}
The MOOC M4D (Mobiles for Development) was a six weeks course from $2^{\text {nd }}$ October- $16^{\text {th }}$ November,2013 on advances of mobile services in many sectors that are vital to human development, such as education, health and agriculture and access to credit and finance. As a librarian the author is interested to know about the teaching/learning process within a MOOC because of reasons that MOOC is an increasingly growing phenomenon and libraries have potential to support MOOCs. The author shares her personal experiences and observations as a MOOC student and presents a short overview of MOOC M4D course, specifically on core issues covered in the course lectures.
\end{abstract}

Index Terms-MOOC, Mobiles for Development, MOOC consortia, Mobile ecosystem, m-learning

\section{BACKGROUND AND COURSE DESIGN}

MOOC stands for Massive Open Online Course designed for anyone to participate without background knowledge or skills, fee and admission process; the only requirement is a username and password to log into the site. The term was originally coined by Dave Cormier along with Bryan Alexander in the year 2008; a close view of literature reveals that George Siemens and Stephen Downes also credited as MOOC pioneers in its current form; the year 2012 brought considerable recognition for MOOC and huge media attention throughout the world. The New York Times termed 2012 the 'Year of the MOOC' and describes MOOC as free, credit less and massive online course [2]. MOOCs have been self-paced class, therefore called asynchronous, since one doesn't have to take it during a specific period. Currently, mobile devices such as smartphones or tablets have a growing role in online learning and society is witnessing unprecedented development on MOOCs.

MOOC M4D was designed for people to have a basic knowledge on mobile ecosystem and anatomy, as well as in-depth understanding of architecting solutions and services that contributes to better living. MOOC M4D (autumn 2013) used open source platform- Sakai LMS (TRUNK - Sakai 2.9.3; Kernel 1.3.3) was constituted with: lectures formatted as short videos combined with forum discussion, online chat session (mentor and learners/peer to peer) and quizzes, primarily useful for those working for advancement in human development through mobile technology. The author summarizes the major themes covered in the course lectures specifically on core issues and submits viewpoints as well as perspectives about the essence and usefulness of this course. The autumn 2013 MOOC M4D course was offered by Indian Institute of Technology, Kanpur, jointly with the Commonwealth of Learning (COL), an inter-governmental agency established in 1987. The course lectures were prepared by a number of faculty from Indian Institute of Technology Kanpur, Indian Institute of Technology, Ropar; Indian Institute of Management, Kolkata and Commonwealth of Learning. The course was categorized under: core topics; specialized modules and additional modules. The core topics focused on education, agriculture and rural banking. The extent of expertise of the instructors reflected through their presentation style, rich content and visually attractive slides which make the reading more fun! There were attachments of all types of format in course lectures (photos, video, scripts, slides etc.) to help learners' understanding, in order to make reading clearer and user friendly. With a consistent structure throughout the duration, the instructors introduced a new lecture series every week. In order to achieve the goals of online instruction it is important to engage both models: learner-centred and instructor-centred. In an online learning context, student activity becomes a key element; it is understood that course team monitored the number of learners visiting the material during the week and as and when it crossed a threshold the next set of materials were uploaded to the site. This process continued for entire duration of this course, and students were graded on the basis of the marks scored in both the quizzes. Each lecture consisted of videos of 10-20 minutes fit into an optimal period of time that students can maintain attention. The flexibility of MOOC M4D attracted a variety of discussions in forums, categorized as technology topics, general discussions and technology application. The course team tracked the access analytics of the learners, and the participants who accessed course lectures at least up to a reasonable proportion considered for participation certificate. The instructors/mentors assessed the competency level using grades in quizzes and eligible participants were issued competency certificates. The certificates were issued by the Center for Continuing Education, IIT Kanpur, co-signed by Commonwealth of Learning.

\section{PARTICIPANTS}

The high level of participation reflected from registration of 115 countries; although a large number of registrants were from developing countries but signed ups from OECD countries such as Canada, France, the Netherlands, New Zealand, UK and USA also significant. It is evident from chat room discussions that a majority of the participants were qualified with formal degrees, also there were young learners as old as 18 years and housewives. 


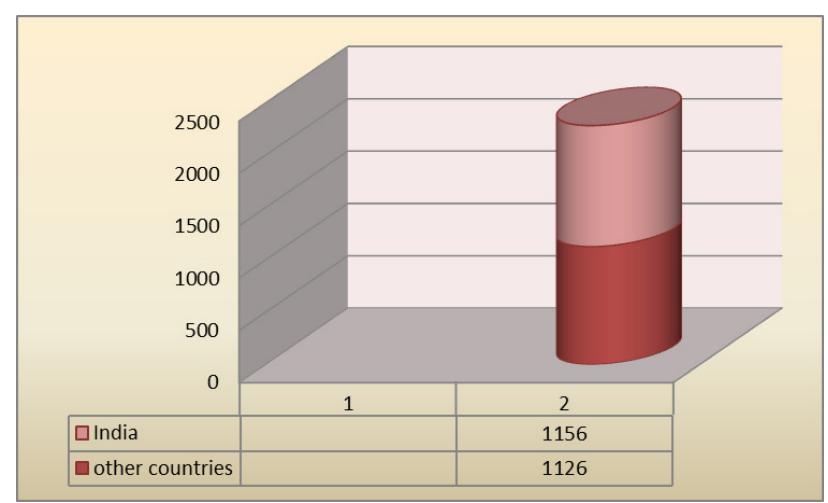

Figure 1. Chart: 1 Statistics of the participants

The course content was designed to enable learners to:

- Recognize frequently used terminology in the mobile arena

- Identify relevant techniques and methods

- Recount key case studies and best practices

- Recognize common policy requirements for successful implementation of mobiles in education, agricultural extension, and rural credit and finance.

Instructors' activities to motivate engaged learning included discussion in chat room, real-time or "live" chat was with the Principal Instructor and mentors, scheduled at regular intervals, weekly e-mail alerts particularly forthcoming events, noteworthy comments on forum entries and an ethos of openness throughout the course, encouraged learners to suggest strategies/ideas on mobiles for human development. The author alludes to the learnercentered pedagogy used by the instructors as a positive contributor to the learning experience.

\section{COURSE LECTURES}

\section{A. Mobile ecosystem and architecting solutions with mobiles}

In a course lecture T.V. Prabhakar from IIT Kanpur, described mobile ecosystem as a set of tools namely mobiles phones, tablets, communication systems, and backend computing systems to support functioning of a mobile; a problem is solved using those tools available within the eco-system. Following are the major challenges and opportunities associated with mobile ecosystem:

TABLE I.

A SUMMARY OF THE CHALLENGES AND OPPORTUNITIES IN A MOBILE ECOSYSTEM

\begin{tabular}{|c|c|}
\hline Challenges & Opportunities \\
\hline $\begin{array}{c}\text { Connectivity: Weak signals, Inter- } \\
\text { mittent, Poor data connectivity }\end{array}$ & Necessity for some sectors \\
\hline $\begin{array}{c}\text { Content Caching is imperative } \\
\text { Power and cost }\end{array}$ & Reachability, Usability and cost \\
\hline $\begin{array}{c}\text { Commodity Hardware: Electronic } \\
\text { Waste is a major problem, Contami- } \\
\text { nation of aquifers }\end{array}$ & Ubiquitous \\
\hline Device Capability & $\begin{array}{c}\text { Multipurpose device } \\
\text { Identity management }\end{array}$ \\
\hline
\end{tabular}

While discussing on Identity management T .V. Prabhakar mentioned that Subscriber Identification Module (SIM) plays an important role in reception and transmis- sion; SIM card coupled with a National Identity number can become a citizen identifier. He further discussed on missed call which is one of the smartest inventions of the common man, it is estimated that companies losses $20 \%$ to $30 \%$ of their revenues due to miscall. He opined that popularity of missed call is due to its simplicity and not about miserliness but easy and time saving. Any mobile device can be connected to a backend to get following applications:

- Interactive voice response

- Speech recognition

- Call/message blast

The Design Pattern of a mobile system includes:

- Content Caching

- Sandbox - its purpose is to isolate the apps, should not harm performance of other apps or snoop on other apps.

Additionally there are Adaptive Apps for changing the behavior dynamically during user interaction for example, Pause of a game/music player while receiving a call, Improve usability and Reduce energy consumption and so on.

\section{B. Smartphone hardware: anatomy of a handset}

Mainak Chaudhuri, IIT Kanpur defines peripheral devices are $\mathrm{I} / \mathrm{O}$ devices through which the end-user interacts with handheld devices; the operating system (OS) needs to have the driver software installed for each such device. Typical peripheral devices are LCD and touchscreen, camera, GPS , Speaker and audio output for headset/earphone ,Microphone, Bluetooth and Wifi. The Processor in a smartphone balances the performance, power consumption, and cost. The ARM-based processors are very common and modern handhelds have multiple application processor. These multi-core application processors deliver excellent performance in low-power resulting in longer battery life. He further emphasizes on ARM TrustZone, which is a hardware-software solution for security in handhelds and protect sensitive information such as various encryption keys. TrustZone hardware allows the application processor to execute in one of the three modes: normal, monitor, and secure; the normal to secure transition happens through monitor. TrustZone software offers a set of secure OS services and the capability to add new user-defined secure services through TrustZone APIs, such as SIM-locking etc. TrustZone hardware adds a "non-secure" or NS bit to every address space to distinguish between secure and non-secure information. The Registers holding encryption keys mapped to secure address space when an application tries to access data residing in a secure address space while the processor is not executing in secure mode, an error is occurred and returned to 'In secure' mode.

\section{Mobile operating systems and Sensors}

In a course lecture Ashis Agarwal from IIT Kanpur mentioned Popular Operating Systems used in mobile devices are: ios, android, widows8, Blackberry OS, FireFox OS, Symbian and Ubunto. It is stated that Android is one of the most popular software stack for mobile devices, based on open source platform, therefore developers do not need any permission for delivering applications. The key applications of Androids are contacts, browsers and 
Operating System is Linux Kernel. Some components of an OS are:

- Kernel - Basic control over hardware devices

- Memory Management- Independent memory for programs

- Multi-tasking

- Disk Access

- User Interface

The different Android versions are named after desserts and sweets and are alphabetically ordered viz. CupCake(1.5), Donut(1.6), Eclair(2.0), Froyo (2.2), Ginger Bread(2.3), Honeycomb(3.0), Ice cream Sand witch (4.0) Jellybean(4.1).He stated Open Handset Alliance (OHA) is an alliance amongst semiconductor companies, software companies and commercialization companies.

Kirti Kumar from IIT Kanpur explained sensors are hardware within a device to feed in data from the physical world; Apps use data from these sensors to perform various functions. The four categories of sensors in mobile phones are: Location Sensors (Wi-Fi, Cell-ID, GPS), Position Sensors (Proximity Sensor- detects the presence of nearby objects), Motion Sensors and Environmental Sensors (measure the ambient conditions of the devicehumidity, temperature, magnetic field and light).In addition to there are miscellaneous sensors viz. Camera, microphone etc.

\section{Cloud Computing: characteristics and classification}

Balwinder Sodhi, at IIT Ropar defines Cloud computing is a computing on remote servers (instead of lo$\mathrm{cal} /$ personal computer); typically hosted on the Internet and exhibits some special properties, for example computing capabilities available on-demand typically in utilities model (pay per use) etc. There are five characteristics of clouds as identified by NIST ${ }^{1}$

- Broad network access

- Resource pooling

- On-demand Self-Service

- Rapid elasticity

- Measured service

Sodhi, further mentioned that cloud providers are of two types: commercial and open source; the examples of commercial cloud providers are Amazon EC2 (Computing), S3 (Storage), Microsoft Azure (Computing and Storage), Google AppEngine (Computing), BigTable (Storage), Salesforce, IBM, Yahoo etc.

The Open Source cloud providers include: OpenNebula, Nimbus, Eucalyptus ,OpenStack , CloudStack , AppScale etc.

Service model based cloud computing are three types:

- Infrastructure as a Service (IaaS), e.g., AWS EC2

- Platform as a Service (PaaS), e.g., Google App Engine

- Software as a Service (SaaS), e.g., Salesforce.com applications run on a cloud infrastructure and accessible from various client devices

\footnotetext{
${ }^{1}$ http://www.nist.gov/itl/cloud/upload/cloud-def-v15.pdf
}

Deployment model based cloud depends on how the cloud is setup/deployed

- Private- Operated by and for an individual entity

- Public - Available to general public like a utility

- Hybrid - Private and public connected together

- Community - Setup by and for a group having shared goals

\section{E. Mobile wireless communications}

Aditya Jagannathan from IIT Kanpur, stated that channel for mobile communication is the air medium and multiple users can simultaneously transmit over the air medium. The Multiple Access Technologies are categorized as:

- FDMA - Frequency Division for Multiple Access

- TDMA - Time Division for Multiple Access.

He further informed GSM (Global System for Mobile Communication) is developed by ETSI (European Telecommunication Standards Institute) is a robust digital cellular standard and an integrated voice-data service that provides a number of services beyond voice.

While discussing on 'Wireless Channel Fading' he mentioned that wireless signal can reach the receiver via direct and multiple scattered paths known as Multipath Propagation. The interference, amplifying or attenuating the signal power is termed as fading and a strong destructive interference is referred as a deep fade.

Under this title 'No Grid Power, No Internet? No Problem for this mobile classroom' Ricky Cheng and V Balaji from Commonwealth Of Learning, demonstrated APTUS - Classroom without walls. It was stated that APATUS device is made up of two devices:

1. A Mini PC acts as a server to host digital learning materials and content or entire learning management systems like Moodle.

2. A Wireless Router acts as a mini "cell tower" to create a Local Area Network which can help connect students' Tablets or phones and acts as power source for Mini PC.

APATUS can store and distribute hundreds of Textbooks can be used for Accessing learning resources, software applications, and material that are available on the internet even when connectivity does not exist. It can be used as a mobile library that downloads and stores learning materials to be accessed offline. Router can be charged easily even by a solar charger.

\section{F. Mobiles in Education:}

Aga Palas from Commonwealth of Learning talked on Educational Mobile Apps; she opined that there is a plethora of apps available for mobiles, among these Games is the most popular apps in the mobile world today. The latest statistics indicates that there are 51 million smartphone users in urban India - an $89 \%$ increase from 2012. As far as the apps, the five categories dominating in India include cricket, music, news, videos and games. The Facebook, Google and Whatsapp lead in terms of the social network apps. She pointed out that although mobile apps in on rise but there is slow adoption of mobile educational apps, especially in formalized teaching. One of the significant reasons is mobile apps are designed to be a personal tool, therefore a bit challenging for the teacher to select an appropriate app and incorporate it in curricular 
activities. Additionally majority of the apps are not designed by expert instructional designers aiming at any specific learning outcome. She cautioned that educators, designers and other users should scrupulously evaluate mobile apps before incorporating them into their learning or teaching practice and proposed few app assessment criteria.

Implementing Successful Mobile Learning and by M. Ally from Commonwealth Of Learning mentioned various Strategies for Successful Implementation of Mobile learning and identified following tutoring Skills of Teachers in Mobile Learning:

- Manage the learning process

- Facilitate learning

- Motivate learners

- Moderate synchronous and asynchronous sessions

- Solve content problems

- Conduct formative evaluation of learning materials

- Evaluate learner performance

- Model and enforce appropriate ethical behavior

Ally, M emphasized that to be successful in mobile learning must be learner-centered and interactive. In another lecture entitled "Future of Education with Mobile Learning" Ally mentioned Open educational resources (OER) are learning materials that are freely available for use, remixing and redistribution; the benefits of OER as follows:

- Low cost to learners

- Social justice: Access by anyone

- Prevent duplication of effort

G. Mobiles on Agriculture: vKVK(Voice Krishi Vigyan Kendra)

vKVK(Voice Krishi Vigyan Kendra)is using Opensource platforms as follows:

- FreeSwitch - virtual telephone exchange

- Plivo - for programming Voice Applications

- Drupal - Php Based Content Management System,

- using MySQL as a database engine

KVKs are considered as reliable source of information \& experts get recognition among farmers, KVK experts are easily approachable and information sent through $\mathrm{vKVK}$ becomes a part of agropedia library and is updated on the site. vKVK is using call blasts application to make better informed agricultural community.

\section{$H$. Content delivery through mobile}

The lecture delivered by T.V. Prabhakar entitled "Madhyam: a low -cost and scalable model for educational content distribution in intermittent connectivity environments" is based on a model for educational content distribution in network-challenged environments, which is implemented and feasibility study completed to deploy in real scenarios. Prabhakar stated 'Madhyam' is about tolerance to intermittent connectivity mechanisms to control the flow of content in the system and mechanisms to analyse the usage of system. According to Prabhakar the processing power and storage capacity of inexpensive commodity hardware can easily run the proposed system. Madhyam's objectives are to:

- Reach learning material to the student

- Help in Decision making

- Reduce administrative and setup costs

The network connections required for this model are as follows:

- Content Repository and delivery point connected by a network which can be intermittent

- Delivery Point and Student's tablet connected through a local Wifi connection

The content repository manages and hosts the content; deliver the content to delivery point

And host the analytics. The Analytics monitored the content usage at the mobile level; content design at the repository (nation/state) level; attendance, and learning issues at the local(school) level.

\section{Miscellaneous topics:}

An interesting lecture by Amitabha Mukherjee from IIT Kanpur on Wikipedia explained the structure and interface of online encyclopaedia managed by anonymous editors which is completely user contributed and available in 243 languages. It's an open source and majority of the contributors are amateurs rather than experts; in spite of that Wikipedia is considered a reliable source. The featured articles are put on the cover page and star is an indicator that an article is of highest category.; unlike the traditional encyclopaedia wiki has news section, the news items are created by editors are voted and placed according to the rank; Wikipedia also includes 'Did you know' column. The English Wikipedia contains highest number of articles over 4 million. The beauty of the Wikipedia is that anybody can log into the site and edit to revert the change; reverting edit is a very common feature in Wikipedia. It is estimated that one fifth of the wiki edits are reverted. He opined that Wikipedia ensures a certain level of standard and is considered as good as other standard online encyclopaedias. In order to maintain a certain amount of standard it is necessary that the statement submitted in Wikipedia should be verifiable against reliable sources (references).

T.V.Prabhakar, IIT Kanpur discussed on Semantic search, he said search engine doesn't search the web in real time instead search an index of the web; the crawling spiders(search engine) searches the documents for the occurrence of the "search string" - substring search, once retrieved the documents is ranked through a critical and complex process. Google uses $200+$ factors for ranking a document.

\section{OBSERVATIONS AND CONCLUSIONS}

Although MOOCs have huge social implications for access to higher education in both the developed and developing countries but practice and potential of MOOCs within education systems in India is still in its nascent stage. The current trends in US universities is forming MOOC consortia and develop partnership with leading international institutions to impart quality education. Some of the universities are also being approached as potential partners to extend the reach and offerings of existing MOOC providers. The MOOC M4D course was a 


\section{REPORT}

\section{MOOC M4D: AN OVERVIEW AND LEARNER'S VIEWPOINT ON AUTUMN 2013 COURSE}

collaborative effort of two leading institutions namely, IIT Kanpur and commonwealth of Learning. The course was a perfect balance between forum posts; listen to course lectures and chat room discussions with instructors and other registered participants. The diversity in the program meant for the participants with diverse background who can fulfil his or her expectations with worthwhile course content. In fact, the course overcomes the challenge of reaching massive audiences with diverse nationalities, backgrounds, abilities and interests. The following are the observations:

- A good initiative with well-organized themes.

- Wide ranging discussions with great number of topics that are covered.

- Sufficient opportunities to network/ express ideas viz. profile information, submit on the wall, feedback at the end of the program etc.

- A large number of participants from developing countries specially from India- one key concern for countries like India is to harness the rapid spread of mobile technology to generate dividends in human development

- The concluding talk on Gandhiji's pedagogy is relevant and applicable.

- The contact information of a large numbers of experts working in mobile arena is very useful.

Fulfilling current educational needs of the online learners and the advances of MOOC like courses require major changes in the traditional roles of libraries. In US public libraries are supporting the MOOC students with internet facilities, technology support and resources. Schwartz (2013) [1] mentioned "There are multiple potential roles for libraries in the MOOC development, support, assessment, and preservation process..... One major, and comparatively mature, role for libraries is in helping faculty ensure the materials they use to create their MOOC presentations and to assign as readings are not going to get them or their institutions into trouble. Faculty members are increasingly used to turning to the library for help with copyright, so in early discussions around making MOOCs work, the library should be front and center".

\section{REFERENCES}

[1] Schwartz, M (2013) Massive Open Opportunity: Supporting MOOCs in Public and Academic Libraries, http://1j.libraryjournal.com/2013/05/library-services/massiveopen-opportunity-supporting-moocs/

[2] New York Times, The Year of the MOOC, 2 November 2012. http://www.nytimes.com/2012/11/04/education/edlife/massiveopen-online-courses-are-multiplying-at-a-rapid-pace.html? $\mathrm{r}=0$

[3] MOOC M4D: https://www.m4d-mooc.org/portal

[4] M4D Course brochure: http://m4d.colfinder.org/sites/default/ files/M4DCourseBrochure.pdf

\section{AUTHOR}

Maitrayee Ghosh is with Florida State University, Tallahassee, FL, USA (maitrayee.ghose@gmail.com).

Submitted 17 December 2013. Published as re-submitted by the author 05 January 2013. 